\title{
Antidepressant use during pregnancy and psychiatric disorders in offspring: Danish nationwide register based cohort study
}

\author{
Xiaoqin Liu, ${ }^{1}$ Esben Agerbo, ${ }^{1,2,3}$ Katja G Ingstrup, ${ }^{1}$ Katherine Musliner, ${ }^{1,3}$ \\ Samantha Meltzer-Brody, ${ }^{4}$ Veerle Bergink, ${ }^{1,5}$ Trine Munk-Olsen, ${ }^{1}$
}

${ }^{1}$ National Center for Register-

based Research, Aarhus

University, Aarhus, Denmark

${ }^{2}$ CIRRAU-Centre for Integrated

Register-based Research,

Aarhus University, Aarhus,

Denmark

${ }^{3}$ Lundbeck Foundation Initiative

for Integrative Psychiatric

Research, iPSYCH, Denmark

${ }^{4}$ Department of Psychiatry,

University of North Carolina at

Chapel Hill School of Medicine,

Chapel Hill, NC, USA

${ }^{5}$ Department of Psychiatry,

Erasmus Medical Centre,

Rotterdam, Netherlands

Correspondence to: X Liu

lxq@econ.au.dk

Additional material is published online only. To view please visit

the journal online.

Cite this as: BMJ 2017;358:j3668 http://dx.doi.org/10.1136/bmj.j3668

Accepted: 19 July 2017

\section{ABSTRACT}

OBJECTIVE

To investigate the association between in utero exposure to antidepressants and risk of psychiatric disorders.

DESIGN

Population based cohort study.

SETTING

Danish national registers.

PARTICIPANTS

905383 liveborn singletons born during 1998 -

2012 in Denmark and followed from birth until July 2014, death, emigration, or date of first psychiatric diagnosis, whichever came first. The children were followed for a maximum of 16.5 years and contributed $8.1 \times 10^{6}$ person years at risk.

EXPOSURES FOR OBSERVATIONAL STUDIES

Children were categorised into four groups

according to maternal antidepressant use within two years before and during pregnancy: unexposed, antidepressant discontinuation (use before but not during pregnancy), antidepressant continuation (use both before and during pregnancy), and new user (use only during pregnancy).

MAIN OUTCOME MEASURE

First psychiatric diagnosis in children, defined as first day of inpatient or outpatient treatment for psychiatric disorders. Hazard ratios of psychiatric disorders were estimated using Cox regression models.

RESULTS

Overall, psychiatric disorders were diagnosed in 32400 children. The adjusted 15 year cumulative incidence of psychiatric disorders was 8.0\% (95\%

\section{WHAT IS ALREADY KNOWN ON THIS TOPIC}

Several studies have linked selective serotonin reuptake inhibitor use during pregnancy to autism spectrum disorder in offspring, although results have been conflicting

The potential explanation for this association is that selective serotonin reuptake inhibitors cross the placental barrier and affect the development of the fetal brain

If this holds true, in utero exposure to selective serotonin reuptake inhibitor and other classes of antidepressants may increase risk for various psychiatric disorders besides autism spectrum disorder

\section{WHAT THIS STUDY ADDS}

Antidepressant use during pregnancy was associated with increased risk for various diagnostic groups of psychiatric disorders in offspring

The observed associations may be attributable to the severity of underlying maternal psychiatric disorders in combination with in utero antidepressant exposure confidence interval $7.9 \%$ to $8.2 \%$ ) in the unexposed group, $11.5 \%$ (10.3\% to $12.9 \%$ ) in the antidepressant discontinuation group, $13.6 \%$ (11.3\% to $16.3 \%)$ in the continuation group, and $14.5 \%(10.5 \%$ to $19.8 \%$ in the new user group. The antidepressant continuation group had an increased risk of psychiatric disorders (hazard ratio 1.27, 1.17 to 1.38), compared with the discontinuation group.

\section{CONCLUSIONS}

In utero exposure to antidepressants was associated with increased risk of psychiatric disorders. The association may be attributable to the severity of underlying maternal disorders in combination with antidepressant exposure in utero. The findings suggest that focusing solely on a single psychiatric disorder among offspring in studies of in utero antidepressant exposure may be too restrictive.

\section{Introduction}

Antidepressants have been increasingly used during pregnancy in the past few decades, ${ }^{1}$ with approximately $2-8 \%$ of pregnant women receiving this treatment. ${ }^{1-7}$ Selective serotonin reuptake inhibitors (SSRIs) are the most commonly prescribed antidepressants. ${ }^{16}$ Although most studies on the safety of SSRI treatment during pregnancy are reassuring, ${ }^{8-11}$ some recent studies have raised concerns about behavioural problems in the offspring. ${ }^{212-17}$

So far, population based studies of long term neurodevelopmental outcomes have focused primarily on autism spectrum disorder in children exposed to SSRIs in utero. Some studies reported an increased risk, ${ }^{13-16}$ whereas others suggested no association. ${ }^{2} 379121819$ The magnitude of the relative risk of autism spectrum disorder for in utero exposure to SSRIs compared with non-exposure ranges from 0.83 to 3.34. ${ }^{2} 37912-161819$ Findings on in utero exposure to SSRIs and attention-deficit/hyperactivity disorder (ADHD) are also contradictory; in utero SSRI exposure was associated with increased risk of ADHD in one study, ${ }^{12}$ but not in others. ${ }^{219-21}$ The underlying mechanism linking in utero SSRI exposure and behavioural problems is unclear; however, one theory posits that SSRIs cross the placental barrier and affect fetal brain development. ${ }^{22-26}$ If this theory holds true, in utero SSRI exposure may increase risks of other psychiatric disorders as well as autism spectrum disorder and ADHD. This assumption is supported by two recent studies investigating other diagnostic groups of psychiatric disorders in children and adolescents besides autism spectrum disorder and ADHD, including depression, anxiety, neurobehavioural and social development, and speech, scholastic, and motor disorders. ${ }^{217}$ Malm et al found an increased 
risk of depression but not anxiety, autism spectrum disorder, or ADHD in children exposed to SSRIs in utero, ${ }^{2}$ and Brown et al suggested that in utero exposure to SSRIs was associated with increased risks of speech and language disorder. ${ }^{17}$ However, these studies did not investigate the overall risk of psychiatric disorders, which is important because differentiating between overlapping symptoms and diagnosing specific disorders are challenging in children and adolescents. Focusing solely on a specific disorder may be too restrictive. Moreover, as serotonin norepinephrine reuptake inhibitors and some tricyclic antidepressants may share a serotonergic component with SSRIs and have a serotonin inhibition effect, ${ }^{27}$ similar effects should be observed with other classes of antidepressants as well as SSRIs. Two studies suggested that risk of autism spectrum disorder in the offspring did not vary by classes of antidepressants. ${ }^{1516}$ However, existing studies on the association between prenatal exposure to non-SSRI antidepressants and psychiatric disorders in offspring had limited numbers of mothers treated with non-SSRI antidepressants,, 37131516182021 preventing in-depth analysis. Additionally, questions about the timing of antidepressant treatment (first, second, or third trimester) and associations with various psychiatric disorders in the exposed offspring have remained unanswered. In summary, gaps remain in the knowledge on antidepressant use during pregnancy and risk of psychiatric disorders in the offspring.

We hypothesised that if in utero exposure to antidepressants affected fetal brain development, such exposure may increase the risk for various psychiatric disorders. We aimed to investigate the association between in utero antidepressant exposure and overall risk of psychiatric disorders. Additionally, we examined risks of individual disorder subcategories including autism spectrum disorder, mood disorder, somatoform disorder, behavioural disorder, and mental retardation, accounting for the timing and duration of treatment as well as the class of antidepressant.

\section{Methods}

\section{Study population}

We did a population based cohort study using data from Danish national registers building on the data from the Danish Civil Registration System. ${ }^{28}$ All live births and residents in Denmark are assigned a unique identification number that permits linkage of individual level data. We identified all liveborn singletons during

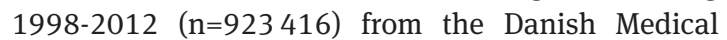
Birth Registry. ${ }^{29}$ We excluded 6569 children who had missing or likely errors in gestational age $(<154$ or $>315$ days) and 1719 children with chromosomal abnormalities (ICD-10 (international classification of diseases, 10th revision) codes Q90-Q99) identified from the Danish National Patient Register. ${ }^{30}$ We further excluded 9745 children with missing links to their fathers. We included a total of 905383 singletons born to 548885 mothers (fig 1).

\section{Antidepressant use}

Information on antidepressant use came from the Danish National Prescription Registry. ${ }^{31}$ This register covers all prescriptions dispensed in Denmark since 1995. It contains the anatomical therapeutic chemical (ATC) classification codes, the number of defined daily doses per package, the number of packages dispensed, and the dispensing date. The ATC code for SSRIs was N06AB, and those for non-SSRI antidepressants were N06AA, N06AF, N06AG, and N06AX (see supplementary table A for detailed ATC codes). We used the dispensing date to indicate the start of antidepressant use. We defined antidepressant use during pregnancy as a prescription dispensed on any date from one month before pregnancy until delivery. We used the first day of the mother's last menstrual period to indicate the start of pregnancy. To capture recent episodes in the mothers, we included antidepressant prescriptions dispensed from two years before pregnancy to delivery. We categorised the children into four mutually exclusive groups according

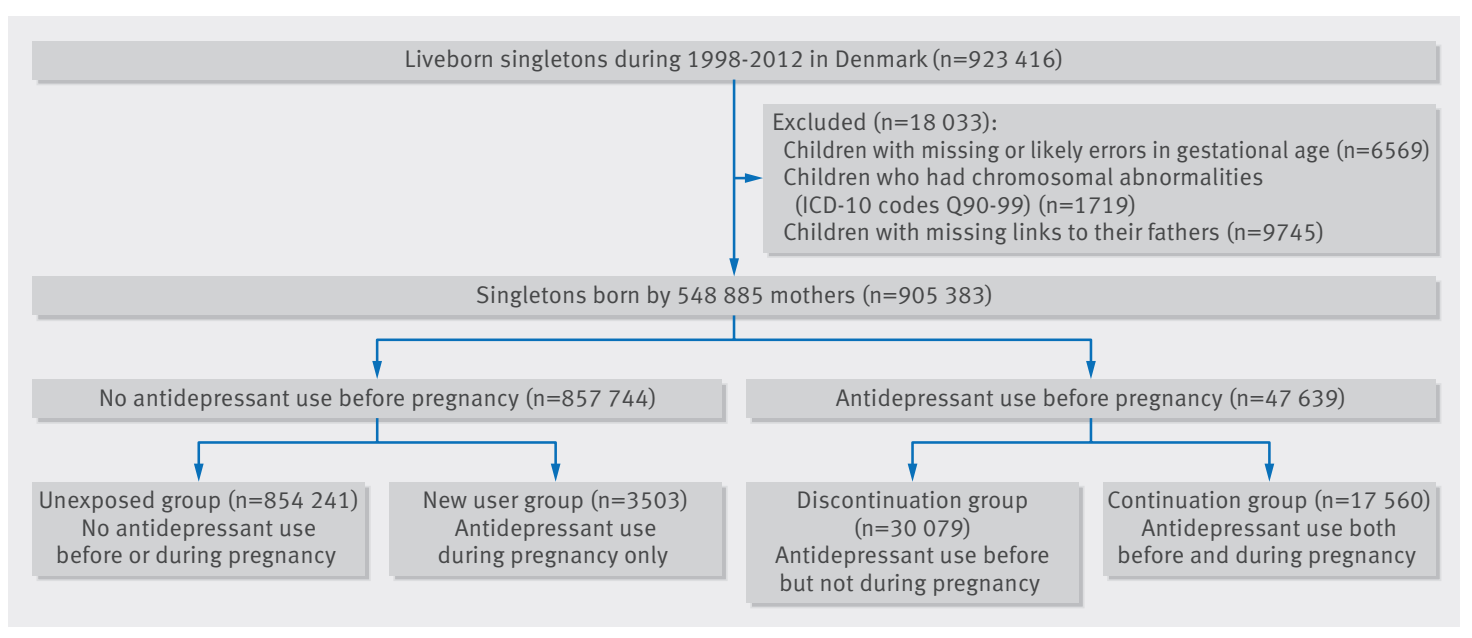

Fig 1 | Flowchart showing identification of study population. Antidepressant use before pregnancy was defined as prescription on any date between two years and one month before pregnancy. Antidepressant use during pregnancy was defined as prescription dispensed on any date from one month before pregnancy until delivery 
to dispensing of an antidepressant prescription to the mother from two years to one month before pregnancy (hereinafter referred to as before pregnancy) and during pregnancy (fig 1). The unexposed group had no maternal antidepressant use before or during pregnancy, the discontinuation group had use before but not during pregnancy, the continuation group had use both before and during pregnancy, and the new user group had use only during pregnancy.

We calculated the number of days exposed per prescription by multiplying the number of defined daily doses per package by the number of packages dispensed. We calculated the exact number of days exposed by adding the durations of all antidepressant prescriptions.

\section{Primary and secondary outcomes: any psychiatric disorders and subcategories of psychiatric disorders}

Information on psychiatric diagnosis came from the Danish Psychiatric Central Research Register. ${ }^{32}$ This register holds information on all inpatient and outpatient psychiatric treatment in Denmark. Our primary outcome was first diagnosis of psychiatric disorders (ICD-10 codes F00-F99) in the offspring. We further specified the following subcategories of psychiatric disorders as secondary outcomes: autism spectrum disorder (F84.0, F84.1, F84.5, F84.8, or F84.9); mood disorder (F30-F39); neurotic, stress related, and somatoform disorder (F40-F48); behavioural and emotional disorder (F90-F98); and mental retardation (F70-F79). We chose the subcategory of autism spectrum disorder on the basis of previous work in this area. The other four categories were defined on the basis of the most commonly observed ICD-10 chapters in our study population.

\section{Potential confounders}

We included the following potential confounders in our analyses on the basis of causal diagrams using directed acyclic graphs: maternal and paternal psychiatric history at delivery, retrieved from the Danish Psychiatric Central Research Register (ICD-8 codes 290351; ICD-10 codes F00-F99); maternal age at delivery (continuous variable); primiparity (yes/no); inpatient and outpatient psychiatric treatment from two years before pregnancy until delivery (yes/no); prescriptions for other psychotropic drugs (ATC codes N05 and N06 excluding N06A) during pregnancy (yes/no); prescriptions for antiepileptic drugs (ATC code N03) during pregnancy (yes/no); number of non-psychiatric hospital visits during pregnancy $(0-1,2-3$, or $\geq 4)$; smoking during pregnancy (yes/no); place of residence (capital or capital suburb, provincial city or town, or rural areas); marital status (married or cohabiting/single, divorced, or widowed); highest education (elementary school/above elementary school); income status (lowest quarter/above lowest quarter); and calendar year of delivery (1998-2002, 2003-07, or 2008-12). Data on these covariates came from the registers mentioned above as well as from Statistics Denmark's registers on socioeconomic status. ${ }^{33}$

\section{Statistical analysis}

We used Stata 13.1 for statistical analyses. We followed each child from birth until July 2014, death, emigration, or the date of first psychiatric diagnosis, whichever came first. For each diagnostic group of psychiatric disorders (secondary outcomes), we defined the date of first diagnosis as the first day of inpatient or outpatient treatment that led to the assignment of the specific diagnosis. We used Cox regression models to calculate hazard ratios with 95\% confidence intervals, with child's age as the time scale. We evaluated proportionality by visually inspecting "log-log" plots. A woman could contribute more than one pregnancy to the analyses. To account for the dependence between siblings, we used the Huber sandwich estimator for correction of standard errors. All P values were based on Wald's tests, and we considered $\mathrm{P}<0.05$ (two sided test) to be statistically significant. About $6.1 \%$ of the values were missing for any of the potential confounders, and we consequently applied 20 imputations by using the Markov Chain Monte Carlo technique for imputing missing values. ${ }^{34}$

We assigned a weight to each child as the estimated inverse probability of being in a certain group. We estimated the weights as predicted values from logistic regression models with adjustment for covariates mentioned above. ${ }^{35}$ We used unadjusted and inverse probability weight adjusted Kaplan-Meier curves to illustrate the cumulative incidence of psychiatric disorders in four groups. To evaluate whether the underlying maternal psychiatric disorders were associated with offspring psychiatric disorders, we calculated the hazard ratios with the unexposed group as the reference group.

\section{Comparisons between antidepressant continuation} and discontinuation groups

To further control for the underlying maternal psychiatric disorders, we did detailed analyses comparing the antidepressant continuation group with the discontinuation group, adjusting for the above mentioned covariates. We estimated the population attributable fraction by using the adjusted hazard ratio of psychiatric disorders in the continuation group versus the discontinuation group and the prevalence of continued antidepressant treatment during pregnancy in the entire population (PE) according to the formula: population attributable fraction $=\mathrm{PE} \times(\mathrm{HR}-1) /(\mathrm{PE} \times(\mathrm{HR}-1)+1)$. The population attributable fraction denotes the proportion of psychiatric cases in our population that could have been prevented if mothers from the continuation group had discontinued antidepressant treatment, assuming the association to be causal and no confounding of the exposure-disease association. ${ }^{36}$

To examine whether the associations between antidepressant exposure and psychiatric disorders depended on the timing of exposure, we divided the exposure window into three groups based on the last menstrual period: first trimester only (one month before pregnancy to 90 days after last menstrual period); 
second or third trimester only (91-180 days after last menstrual period or 181 days after last menstrual period to childbirth); and more than one trimester. We considered a child to be exposed to antidepressants in a specific trimester if the dispensing date fell within the trimester or if the number of days prescribed overlapped that trimester. To study whether the associations varied with different classes of antidepressants, we categorised antidepressant treatment into SSRI monotherapy, non-SSRI antidepressant monotherapy, and both SSRI and non-SSRI antidepressant treatment. To determine whether the associations were modified by the duration of use, we divided the duration of antidepressant use during pregnancy into 90 days or less, 91-180 days, and 181 days or more. We also included the duration as a continuous variable, per 30 day increase, in the models to see whether durationresponse relations existed.

\section{Sensitivity analysis}

To test the robustness of our results, we did seven sensitivity analyses. Firstly, to reduce the misclassification of antidepressant exposure, we restricted our analyses to mothers who filled at least two prescriptions for antidepressants during pregnancy. We did this to exclude mothers who possibly decided not to take antidepressants in pregnancy and hence did not renew their prescriptions. Secondly, to reduce the misclassification of discontinuation of antidepressant use, we redefined antidepressant discontinuation as maternal use of antidepressant from two years to three months before pregnancy but not from three months before pregnancy until delivery. Thirdly, to overcome any concerns about the validity of psychiatric diagnoses, we repeated our analysis by redefining psychiatric disorders in offspring as at least two hospital contacts for psychiatric disorders in the register, thereby defining a stricter phenotype. Fourthly, mothers who had inpatient psychiatric treatment may have more severe symptoms. To further account for the confounding by the underlying indication for treatment, we repeated our analyses by excluding 2644 pregnancies in mothers with any inpatient psychiatric treatment from two years before pregnancy until the delivery. Fifthly, to examine whether the associations between maternal antidepressant use and psychiatric disorders in offspring were confounded by shared environmental or genetic variables, we included comparisons with associations between paternal antidepressant use and psychiatric disorders in offspring. ${ }^{37}$ We further adjusted for maternal antidepressant use during pregnancy (unexposed, discontinuation, continuation, or new user group), paternal age at delivery (continuous variable), and paternal inpatient or outpatient psychiatric treatment from two years before pregnancy to delivery (yes/no). Our working hypothesis was that if any potential effects are due to an intrauterine exposure, maternal antidepressant use during pregnancy should have a greater influence than paternal antidepressant use during the index pregnancy. ${ }^{37}$ Sixthly, to test whether the associations were modified by maternal psychiatric history, we re- peated our analyses stratified by maternal psychiatric history. Finally, we investigated potential sex specificity of associations by inclusion of an interaction term between sex of the child and antidepressant exposure.

\section{Patient involvement}

No patients were involved in setting the research question or the outcome measures, nor were they involved in developing plans for design or implementation of the study. No patients were asked to advise on interpretation or writing up of results. There are no plans to disseminate the results of the research to study patients or the relevant patient community.

\section{Results}

Table 1 lists socioeconomic, psychiatric, and demographic characteristics of the study population. Of the 905383 children, 21063 (2.3\%) were born to mothers who used antidepressants during pregnancy. Approximately $1.8 \% \quad(n=16154)$ of mothers used SSRI monotherapy, 0.4\% $(n=3295)$ used non-SSRI antidepressant monotherapy, and 0.2\% ( $\mathrm{n}=1614)$ used both SSRI and non-SSRI antidepressants (supplementary table A). The children were followed for a maximum of 16.5 years and contributed $8.1 \times 10^{6}$ person years at risk. Overall, 32400 children were diagnosed as having psychiatric disorders. The mean age at first psychiatric diagnosis was 8.5 (SD 3.6) years. The weighted 15 year cumulative incidence of psychiatric disorders in the offspring was $8.0 \%$ (95\% confidence interval $7.9 \%$ to $8.2 \%$ ) for the unexposed group, $11.5 \%$ (10.3\% to $12.9 \%)$ for the discontinuation group, $13.6 \%$ (11.3\% to $16.3 \%)$ for the continuation group, and $14.5 \%$ (10.5\% to $19.8 \%)$ for the new user group (fig 2). We observed increased risks of psychiatric disorders in all three groups of antidepressant users (discontinuation, continuation, and new user groups), compared with the unexposed group (supplementary figure).

\section{Comparisons between antidepressant continuation and discontinuation groups}

The risk for psychiatric disorders among offspring in the continuation group was higher than that in the discontinuation group (hazard ratio 1.27, 95\% confidence interval 1.17 to 1.38 ). This was apparent after adjustment for demographic and psychiatric characteristics of the mothers, including inpatient and outpatient psychiatric treatment and comorbid psychotropic drug use (table 2). The population attributable fraction was $0.5 \%$, indicating that continuous antidepressant use during pregnancy explained $0.5 \%$ of psychiatric cases in the study population, assuming an unconfounded causal association.

Children exposed to antidepressant during the first trimester only had the lowest risk of psychiatric disorders compared with children exposed in the second or third trimester only or exposed during more than one trimester. The risk of psychiatric disorders did not differ between children exposed to SSRI monotherapy and non-SSRI antidepressant 


\begin{tabular}{|c|c|c|c|c|}
\hline Characteristics & $\begin{array}{l}\text { Unexposed group* } \\
(\mathrm{n}=854241)\end{array}$ & $\begin{array}{l}\text { Antidepressant discontinuation } \\
\text { groupt }(n=30079)\end{array}$ & $\begin{array}{l}\text { Continuation group } \neq \\
(\mathrm{n}=17560)\end{array}$ & $\begin{array}{l}\text { New user group } \S \\
(n=3503)\end{array}$ \\
\hline Mean (SD) maternal age at delivery, years & $30.2(4.8)$ & $30.1(5.2)$ & $30.8(5.2)$ & $29.4(5.6)$ \\
\hline Primiparity & $375698(44.0)$ & $13560(45.1)$ & $7914(45.1)$ & $1397(39.9)$ \\
\hline Maternal psychiatric history at delivery & $37410(4.4)$ & $9440(31.4)$ & $8494(48.4)$ & $1168(33.3)$ \\
\hline $\begin{array}{l}\text { Inpatient psychiatric treatment from } 2 \text { years before pregnancy to } \\
\text { delivery }\end{array}$ & $1796(0.2)$ & $1296(4.3)$ & $1348(7.7)$ & $184(5.3)$ \\
\hline $\begin{array}{l}\text { Outpatient psychiatric treatment from } 2 \text { years before pregnancy } \\
\text { to delivery }\end{array}$ & $8939(1.0)$ & $4871(16.2)$ & $5006(28.5)$ & $806(23.0)$ \\
\hline Dispensing of other psychotropic prescriptions during pregnancy & $4816(0.6)$ & $656(2.2)$ & $1140(6.5)$ & $468(13.4)$ \\
\hline Dispensing of antiepileptic prescriptions during pregnancy & $231(<0.1)$ & $34(0.1)$ & $75(0.4)$ & $21(0.6)$ \\
\hline \multicolumn{5}{|l|}{ No of non-psychiatric hospital visits during pregnancy: } \\
\hline $0-1$ & $202879(23.7)$ & $4616(15.3)$ & $2577(14.7)$ & $515(14.7)$ \\
\hline $2-3$ & $428562(50.2)$ & $13349(44.4)$ & $7750(44.1)$ & $1492(42.6)$ \\
\hline$\geq 4$ & $222800(26.1)$ & $12114(40.3)$ & $7233(41.2)$ & $1496(42.7)$ \\
\hline \multicolumn{5}{|l|}{ Maternal smoking during pregnancy: } \\
\hline Yes & $137089(16.0)$ & $8867(29.5)$ & $5325(30.3)$ & $1180(33.7)$ \\
\hline No & $694874(81.3)$ & $20431(67.9)$ & $11743(66.9)$ & $2224(63.5)$ \\
\hline Missing & $22278(2.6)$ & $781(2.6)$ & $492(2.8)$ & $99(2.8)$ \\
\hline \multicolumn{5}{|l|}{ Place of residence at delivery: } \\
\hline Capital or capital suburb & $254423(29.8)$ & $7262(24.1)$ & $4160(23.7)$ & $798(22.8)$ \\
\hline Provincial city or town & $334536(39.2)$ & $12600(41.9)$ & $7679(43.7)$ & $1491(42.6)$ \\
\hline Rural areas & $265173(31.0)$ & $10216(34.0)$ & $5719(32.6)$ & $1212(34.6)$ \\
\hline Missingी & $109(<0.1)$ & $<5(<0.1)$ & $<5(<0.1)$ & $<5(<0.1)$ \\
\hline \multicolumn{5}{|l|}{ Maternal marital status at delivery: } \\
\hline Married or cohabiting & $752769(88.1)$ & $23630(78.6)$ & $13646(77.7)$ & $2673(76.3)$ \\
\hline Single, divorced, or widowed & $101472(11.9)$ & $6449(21.4)$ & $3914(22.3)$ & $830(23.7)$ \\
\hline \multicolumn{5}{|l|}{ Maternal highest education at delivery: } \\
\hline Elementary school & $151931(17.8)$ & 9966 (33.1) & $5262(30.0)$ & $1337(38.2)$ \\
\hline Above elementary school & $671138(78.6)$ & $19547(65.0)$ & $12001(68.3)$ & $1997(57.0)$ \\
\hline Missing & $31172(3.6)$ & $566(1.9)$ & $297(1.7)$ & $169(4.8)$ \\
\hline \multicolumn{5}{|l|}{ Maternal income status at delivery: } \\
\hline Lowest quarter & $141866(16.6)$ & $6534(21.7)$ & $4449(25.3)$ & $752(21.5)$ \\
\hline Above lowest quarter & $712120(83.4)$ & $23544(78.3)$ & $13110(74.7)$ & $2750(78.5)$ \\
\hline Missing & $255(<0.1)$ & $<5(<0.1)$ & $<5(<0.1)$ & $<5(<0.1)$ \\
\hline \multicolumn{5}{|l|}{ Calendar year of delivery: } \\
\hline 1998-2002 & $302651(35.4)$ & $5707(19.0)$ & $2297(13.1)$ & $650(18.6)$ \\
\hline 2003-07 & $287096(33.6)$ & $10578(35.1)$ & $5778(32.9)$ & $1270(36.3)$ \\
\hline $2008-12$ & $264494(31.0)$ & $13794(45.9)$ & $9485(54.0)$ & $1583(45.2)$ \\
\hline Paternal psychiatric history at delivery & $30257(3.5)$ & $2587(8.6)$ & $1676(9.5)$ & $324(9.2)$ \\
\hline \multicolumn{5}{|l|}{ Paternal antidepressant use: } \\
\hline No antidepressant use & $821716(96.2)$ & 27034 (89.9) & $15298(87.1)$ & $3083(88.0)$ \\
\hline Use before but not during pregnancy & $13385(1.6)$ & $1377(4.6)$ & $784(4.5)$ & $161(4.6)$ \\
\hline Use both before and during pregnancy & $11984(1.4)$ & $1098(3.7)$ & $1078(6.1)$ & $152(4.3)$ \\
\hline Use during but not before pregnancy & $7156(0.8)$ & $570(1.9)$ & $400(2.3)$ & $107(3.1)$ \\
\hline \multicolumn{5}{|l|}{ Sex of child: } \\
\hline Male & $438176(51.3)$ & $15553(51.7)$ & $9070(51.7)$ & $1829(52.2)$ \\
\hline Female & $416065(48.7)$ & $14526(48.3)$ & $8490(48.3)$ & $1674(47.8)$ \\
\hline
\end{tabular}

*Children whose mothers did not use antidepressant before or during pregnancy.

tChildren whose mothers used antidepressants before but not during pregnancy.

‡Children whose mothers used antidepressants both before and during pregnancy.

$\S$ Children whose mothers used antidepressants during but not before pregnancy.

१To comply with Danish regulations aimed at safeguarding anonymity of people included in register based research studies, numbers less than 5 cannot be shown.

monotherapy, although statistical precision was low for non-SSRI antidepressant monotherapy. The highest risk was among children whose mothers used both SSRI and non-SSRI antidepressants (hazard ratio 1.72, 1.40 to 2.11). We found no marked differences in the hazard ratios of psychiatric disorders among the most commonly prescribed SSRIs, although the statistical precision was low for paroxetine and escitalopram. The hazard ratios were 1.32 (1.15 to 1.51) for citalopram monotherapy, 1.26 (1.05 to 1.51) for sertraline, 1.22 (1.02 to 1.45) for fluoxetine, 1.17 (0.94 to 1.46) for paroxetine, and 1.14 (0.78 to 1.68) for escitalopram, compared with the discontinuation group. For different durations of antidepressant use, a higher risk was seen among children whose mothers took antidepressants for more than 180 days (hazard ratio 1.40, 1.26 to 1.56) (table 2).

We also saw increased risks in the continuation group compared with the discontinuation group for autism spectrum disorder (hazard ratio 1.23, 1.01 to 1.51), mood disorder (2.76, 1.59 to 4.78$)$, somatoform disorder (1.62, 1.36 to 1.94$)$, and behavioural disorder (1.13, 1.01 to 1.27$)$, but not mental retardation (1.21, 0.78 to 1.90 ) (table 3 ). The hazard ratios of specific 


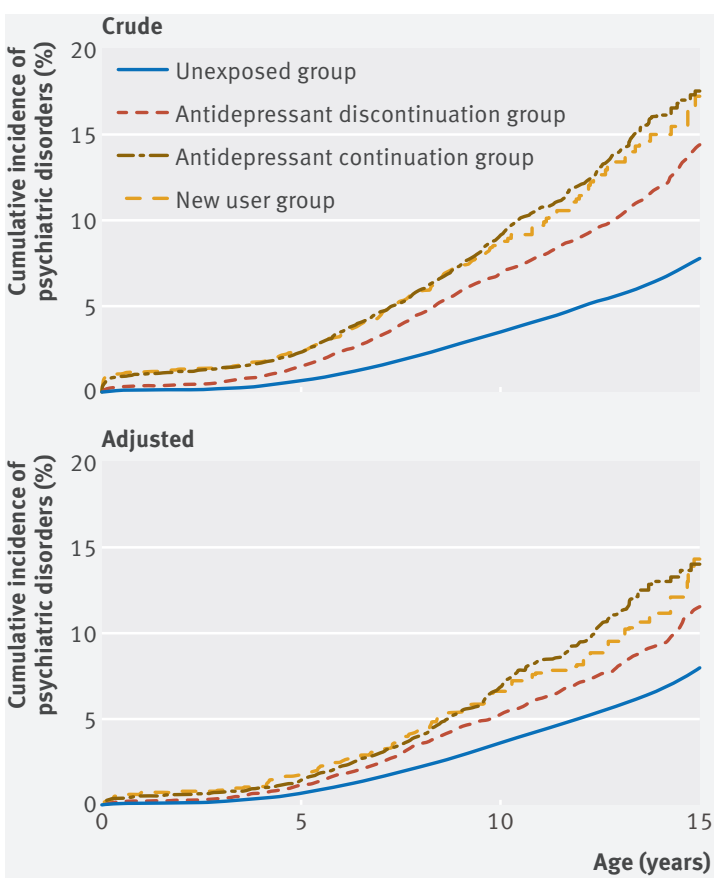

Fig 2 | Crude and adjusted Kaplan-Meier curves illustrating cumulative incidence of any psychiatric disorders in children according to maternal antidepressant use before and during pregnancy $(n=905383)$. Unexposed group: children whose mothers did not use antidepressant before or during pregnancy. Antidepressant discontinuation group: children whose mothers used antidepressants before but not during pregnancy. Antidepressant continuation group: children whose mothers used antidepressants both before and during pregnancy. New user group: children whose mothers used antidepressants during but not before pregnancy. Adjusted for maternal age at delivery, primiparity, maternal psychiatric history at delivery, inpatient and outpatient psychiatric treatment from two years before pregnancy until delivery, dispensing of other psychotropic prescriptions during pregnancy, dispensing of antiepileptic prescriptions during pregnancy, number of non-psychiatric hospital visits during pregnancy, smoking during pregnancy, place of residence, marital status, highest education, income, calendar year of delivery, and paternal psychiatric history at time of delivery

diagnostic groups of psychiatric disorders according to timing, class, and duration of antidepressant use are shown in supplementary tables B-D.

\section{Sensitivity analyses}

Among mothers who continued antidepressant use during pregnancy, $74.4 \%(n=13056)$ received two or more prescriptions. Redefining antidepressant exposure as two prescriptions gave a hazard ratio of psychiatric disorders for the continuation group of 1.33 (1.21 to 1.46), compared with the discontinuation group (supplementary table E). Sensitivity analyses showed similar results when we redefined antidepressant discontinuation as use of antidepressants before pregnancy with no use from three months before pregnancy until delivery or excluded pregnancies in mothers with inpatient psychiatric treatment from two years before pregnancy until delivery (supplementary tables $F$ and $G$ ). When we redefined psychiatric disorders in offspring as at least two hospital contacts for psychiatric disorders, 17735 children were categorised as having psychiatric disorders. The result remained unchanged, with a hazard ratio of 1.20 (1.06 to 1.35) for the continuation group versus the discontinuation group. We found that paternal antidepressant use during pregnancy was associated with overall psychiatric, somatoform, and behavioural disorders, but not other diagnostic groups of psychiatric disorders. The associations between paternal antidepressant use and psychiatric disorders in offspring were weaker than the associations between maternal antidepressant use and psychiatric disorders in offspring (supplementary figure). The associations between in utero antidepressant exposure and psychiatric disorders were slightly but not statistically significantly higher among children born to mothers with a psychiatric history than in children born to mothers with no psychiatric history (supplementary table $\mathrm{H}$ ). We found no evidence to suggest that the associations between in utero antidepressant exposure and psychiatric disorders were modified by sex of the child (all $\mathrm{P}$ values for interaction were greater than 0.2 ).

\section{Discussion}

We observed increased risks for psychiatric disorders among children of mothers who used antidepressants before and/or during pregnancy, compared with mothers with no record of antidepressant use. Furthermore, we observed an increased risk of psychiatric disorders in children whose mothers continued antidepressant use during pregnancy, compared with mothers who discontinued. These associations could be attributable to the severity of the underlying maternal disorders in combination with in utero antidepressant exposure. ${ }^{5}$

We found that children whose mothers discontinued antidepressant use also had an increased risk of psychiatric disorders. This association is most likely driven by the underlying maternal psychiatric disorders, which may be transmitted to offspring via shared genetic susceptibility, environmental stress, and/or parenting practices. ${ }^{383940}$ We did observe an increased risk of psychiatric disorders among children exposed to antidepressants in utero. We speculated that this increased risk could be due to the severity of underlying maternal psychiatric disorders because mothers with severe symptoms are more likely to continue treatment during pregnancy. ${ }^{5}$

We tried to account for the underlying disorders by adjusting for various psychiatric variables including maternal and paternal psychiatric history, dispensing of prescriptions for antiepileptic and other psychotropic drugs during pregnancy, and inpatient and outpatient psychiatric treatment before pregnancy. After adjustment, the increased risk of psychiatric disorders 


\begin{tabular}{|c|c|c|c|c|c|}
\hline Antidepressant use during pregnancy & Cases & No & Person years & Crude hazard ratio & Adjusted hazard ratio $(95 \% \mathrm{Cl})^{*}$ \\
\hline Antidepressant discontinuation groupt & 1417 & 30079 & $2.2 \times 1^{0} 5$ & 1 & 1 (reference) \\
\hline Antidepressant continuation group $\ddagger$ & 944 & 17560 & $1.2 \times 1^{0} 5$ & 1.40 & $1.27(1.17$ to 1.38$)$ \\
\hline \multicolumn{6}{|l|}{ Timing of antidepressant use: } \\
\hline Use in first trimester only & 249 & 3772 & $3.0 \times 1^{\circ} 4$ & 1.21 & $1.16(1.01$ to 1.33$)$ \\
\hline Use in second or third trimester only & 36 & 575 & $3.8 \times 1^{0} 3$ & 1.61 & $1.42(1.02$ to 1.97$)$ \\
\hline Use in more than one trimester & 659 & 13213 & $8.1 \times 1^{0} 4$ & 1.48 & $1.32(1.20$ to 1.45$)$ \\
\hline \multicolumn{6}{|l|}{ Class of antidepressant use: } \\
\hline SSRI monotherapy & 722 & 13501 & $9.0 \times 1^{0} 4$ & 1.36 & $1.25(1.14$ to 1.37$)$ \\
\hline Non-SSRI antidepressant monotherapy & 119 & 2610 & $1.6 \times 1^{0} 4$ & 1.26 & $1.15(0.96$ to 1.40$)$ \\
\hline Use both SSRI and non-SSRI antidepressants & 103 & 1449 & $9.0 \times 1^{0} 3$ & 2.05 & $1.72(1.40$ to 2.11$)$ \\
\hline \multicolumn{6}{|l|}{ Duration of antidepressant use: } \\
\hline$\leq 90$ days & 263 & 4075 & $3.2 \times 1^{0} 4$ & 1.20 & $1.15(1.01$ to 1.31$)$ \\
\hline $91-180$ days & 184 & 3504 & $2.3 \times 1^{0} 4$ & 1.32 & $1.18(1.01$ to 1.38$)$ \\
\hline$\geq 181$ days & 497 & 9981 & $6.0 \times 1^{0} 4$ & 1.57 & $1.40(1.26$ to 1.56$)$ \\
\hline Per 30 day increase & - & - & - & 1.03 & $1.02(0.99$ to 1.06$)$ \\
\hline
\end{tabular}

SSRI=selective serotonin reuptake inhibitor.

${ }^{*}$ Adjusted for maternal age at delivery, primiparity, maternal psychiatric history at delivery, inpatient and outpatient psychiatric treatment from 2 years before pregnancy until delivery, dispensing of other psychotropic prescriptions during pregnancy, dispensing of antiepileptic prescriptions during pregnancy, number of non-psychiatric hospital visits during pregnancy, smoking during pregnancy, place of residence, marital status, highest education, income, calendar year of delivery, and paternal psychiatric history at time of delivery.

tChildren whose mothers used antidepressants before but not during pregnancy; used as comparison group for all comparisons.

$\neq$ Children whose mothers used antidepressants both before and during pregnancy.

in offspring remained statistically significant, but the magnitude of the association was attenuated.

In a next step, we repeated all our analyses for paternal antidepressant use during the index pregnancy, with psychiatric disorders in offspring as the outcome. We found that continuous paternal antidepressant use during pregnancy was also associated with slightly increased risk of overall psychiatric disorders in offspring, but a stronger association was observed for maternal antidepressant use. For somatoform disorder, the influence was similar for maternal and paternal antidepressant exposure. For other diagnostic groups of psychiatric disorders, significant differences were observed as the confidence intervals of these estimates did not overlap. The observed differences in results for maternal versus paternal antidepressant use may indicate an intrauterine effect over and beyond any shared genetic, familial, or environmental factors. ${ }^{37}$ Maternal antidepressant use and/or underlying disorders during pregnancy may affect the intrauterine environment and programme fetal neurodevelopment via alterations of maternal hormonal and behavioural responses to stress, placental function and perfusion, fetal hypothalamic-pituitary-adrenal axis, and epigenetic profiles, alone or in combination. ${ }^{41}$

We observed a higher risk among children whose mothers took antidepressants for more than 180 days, meaning that longer exposure periods were associated with higher risks. Similarly, a longer duration of treatment may reflect persistent symptoms in the mothers, and the duration of disorders predicts adverse neurodevelopment of offspring. ${ }^{42}$ We found that the risk of psychiatric disorders in offspring did not vary by class of antidepressants. The highest risk was

\begin{tabular}{|c|c|c|c|c|c|}
\hline Antidepressant use during pregnancy* & Cases & No & Person years & Crude hazard ratio & Adjusted hazard ratio $(95 \% \mathrm{Cl}) \dagger$ \\
\hline \multicolumn{6}{|l|}{ Autism spectrum disorder: } \\
\hline Antidepressant discontinuation group & 257 & 30079 & $2.2 \times 1^{0} 5$ & 1 & 1 (reference) \\
\hline Antidepressant continuation group & 165 & 17560 & $1.2 \times 1^{0} 5$ & 1.32 & $1.23(1.01$ to 1.51$)$ \\
\hline \multicolumn{6}{|l|}{ Mood disorder: } \\
\hline Antidepressant discontinuation group & 26 & 30079 & $2.2 \times 1^{0} 5$ & 1 & 1 (reference) \\
\hline Antidepressant continuation group & 27 & 17560 & $1.2 \times 1^{0} 5$ & 2.72 & $2.76(1.59$ to 4.78$)$ \\
\hline \multicolumn{6}{|c|}{ Neurotic, stress related, and somatoform disorder: } \\
\hline Antidepressant discontinuation group & 276 & 30079 & $2.2 \times 1^{0} 5$ & 1 & 1 (reference) \\
\hline Antidepressant continuation group & 249 & 17560 & $1.2 \times 1^{0} 5$ & 1.92 & $1.62(1.36$ to 1.94$)$ \\
\hline \multicolumn{6}{|l|}{ Behavioural and emotional disorder: } \\
\hline Antidepressant discontinuation group & 789 & 30079 & $2.2 \times 1^{0} 5$ & 1 & 1 (reference \\
\hline Antidepressant continuation group & 470 & 17560 & $1.2 \times 1^{0} 5$ & 1.24 & $1.13(1.01$ to 1.27$)$ \\
\hline \multicolumn{6}{|l|}{ Mental retardation: } \\
\hline Antidepressant discontinuation group & 53 & 30079 & $2.2 \times 1^{0} 5$ & 1 & 1 (reference) \\
\hline Antidepressant continuation group & 33 & 17560 & $1.2 \times 1^{0} 5$ & 1.25 & $1.21(0.78$ to 1.90$)$ \\
\hline
\end{tabular}

*Antidepressant discontinuation group=children whose mothers used antidepressants before but not during pregnancy; antidepressant continuation group=children whose mothers used antidepressants both before and during pregnancy.

tAdjusted for maternal age at delivery, primiparity, maternal psychiatric history at delivery, inpatient and outpatient psychiatric treatment from 2 years before pregnancy until delivery, dispensing of other psychotropic prescriptions during pregnancy, dispensing of antiepileptic prescriptions during pregnancy, number of non-psychiatric hospital visits during pregnancy, smoking during pregnancy, place of residence, marital status, highest education, income, calendar year of delivery, and paternal psychiatric history at time of delivery. 
observed among children exposed to both SSRI and non-SSRI antidepressants, indicating that the mothers were treated with polypharmacy or switched drugs. Mothers may be treated with polypharmacy or switch drugs because of limited/absent treatment response or psychiatric comorbidities, ${ }^{43} 44$ consequently exposing the fetus to insufficiently managed underlying maternal disorders. We also found a higher risk among children whose mothers used antidepressants in the second or third trimester only and for whom the drugs were prescribed across more than one trimester. We hypothesise that these findings can also be attributed to the severity of maternal symptoms, as mothers may restart antidepressants in the second and third trimester because of severe psychiatric symptoms or relapse. ${ }^{43}$

\section{Findings on specific diagnostic groups of psychiatric disorders}

In this study, in utero exposure to antidepressants was associated with a marginally elevated risk of autism spectrum disorder and behavioural disorder including ADHD.Studiesonthistopicareinconsistent, ${ }^{237912-1618-21}$ which makes treatment decisions challenging for individual women and their treating physicians. Recently, two studies reported an increased risk of autism spectrum disorder after exposure to antidepressant in utero in the population cohort but not in the sibling comparison. ${ }^{719}$ Although a sibling design provides one efficient approach to control for time stable familial factors, sibling comparisons are subject to several limitations including highly selected populations, non-shared confounding bias, and by default smaller sample size. ${ }^{45}$ Thereby, any interpretation based solely on sibling comparisons is complicated. On the basis of our findings and previous findings, the observed associations of in utero antidepressant exposure with autism spectrum disorder and behavioural disorder are modest, if they exist.

We found a strong association between maternal antidepressant use during pregnancy and mood disorder in the offspring. This is in line with a recent study using Finnish national registers. ${ }^{2}$ Despite the association observed in two large independent datasets, no evidence exists in humans to show that antidepressant use causes vulnerability for mood disorder in offspring. In contrast, experimental animal studies showed that prenatal exposure to SSRIs led to increased depression and anxiety-like behaviour, decreased sociability, and impaired learning. ${ }^{232546}$ Moreover, in animal models, intrauterine SSRI exposure altered hormonal and behavioural responses to stress and induced epigenetic changes. ${ }^{41}$ The rationale for these results could be the fact that serotonin acts as a trophic factor, particularly in those brain systems that modulate emotional function. ${ }^{41}$ Serotonin has an influence on several developmental processes such as neuronal differentiation, migration, and synaptogenesis. Consequently, early manipulation of the serotonergic system could contribute to emotional disorders in offspring.
Clinical implications: antidepressant continuation or discontinuation during pregnancy?

We found an association between antidepressant use during pregnancy and psychiatric disorders in offspring, and the corresponding population attributable fraction was $0.5 \%$. From a public health perspective, this indicates that $0.5 \%$ of psychiatric disorders in our population could have been prevented if mothers in the antidepressant continuation group had not taken antidepressants during pregnancy, although only if the association is causal and antidepressant exposure is independent from other factors that influence psychiatric disorders. ${ }^{36}$

The decision to discontinue or maintain antidepressant treatment during pregnancy is challenging. Discontinuation of antidepressant treatment can lead to psychiatric episodes with subsequent long lasting adverse effects on both the mother and child. ${ }^{47}$ Conversely, discontinuation of antidepressants might be an option for a subgroup of women. ${ }^{48}$ Decision making tools and treatment algorithms to identify subgroups of women who can be tapered off antidepressants safely are urgently needed. Clinical guidelines are available, including a review from the American Psychiatric Association and the American Association of Obstetricians and Gynecologists. ${ }^{48}$ This review concludes that patients with no or mild depressive symptoms during a six month period may be candidates for discontinuation before conception. In contrast, patients with a history of severe, recurrent depression may not be suitable candidates for drug discontinuation before or during pregnancy. The findings of our study do not change these recommendations. Importantly, any final decision on antidepressant continuation should be individualised and made jointly by health professionals and patients.

\section{Strengths and limitations of study}

We examined a wide range of psychiatric disorders with a large sample size, allowing evaluation of the associations between particular classes of antidepressants and specific diagnostic groups of psychiatric disorders. Information on antidepressant use and psychiatric diagnoses were collected prospectively, which reduces the chance of recall bias. Our study has a follow-up period up to 16.5 years, which enabled us to investigate long term outcomes.

Our study also has limitations. Firstly, we used prescription data to define antidepressant exposure. As some patients who filled prescriptions may not take the antidepressants, we might have misclassified them as exposed. However, compliance with antidepressant treatment during pregnancy is high in Denmark, ${ }^{49}$ so any misclassification is likely to be limited. Furthermore, we obtained similar results when we redefined antidepressant exposure on the basis of two prescriptions. We estimated the duration of antidepressant treatment as the number of defined daily doses, a measure defined by the World Health Organization, relating to the average amount of a drug needed for long term therapeutic use. However, prescribed daily doses 
might differ, ${ }^{50}$ which can lead to potential misclassification of duration of treatment. This misclassification would have biased our estimate of the effect of different durations of treatment on psychiatric disorders in offspring towards each other.

Secondly, we used the date of first psychiatric treatment as first psychiatric diagnosis. Mothers who take antidepressants may be more likely to seek treatment for their children, which could introduce detection bias. However, we investigated several diagnostic groups of psychiatric disorders, some of which have a later onset. ${ }^{51}$ Similar associations were observed irrespective of age at onset. Therefore we doubt that our findings can be ascribed to detection bias. Furthermore, children with one hospital contact for psychiatric disorders may have transient conditions. To overcome this potential misclassification of our outcome, we repeated our analysis by redefining psychiatric disorders in offspring by at least two hospital contacts for psychiatric disorders, but similar results were obtained.

Thirdly, mothers who took antidepressants may have more severe underlying disorders than nonusers. Although we attempted to control for the underlying indication, confounding by severity may still occur. Fourthly, this is an observational study, and unmeasured confounding is always a concern in these types of studies. We were unable to include some important covariates, such as alcohol consumption, which are not available in the registers. Fifthly, although our study has a large sample size, some subgroup analyses are based on quite small numbers of cases. Lastly, although our findings suggest an association between in utero antidepressant exposure and psychiatric disorders in offspring, our results do not confirm causality.

\section{Conclusion}

Antidepressant use during pregnancy was associated with increased risk of psychiatric disorders in offspring. The association may be attributable to the severity of underlying maternal disorders in combination with antidepressant exposure in utero. Our findings suggest that focusing solely on a single psychiatric disorder among offspring in studies of in utero antidepressant exposure may be too restrictive.

Contributors: $X L, E A, V B$, and TMO conceived and designed the study. $\mathrm{XL}$ analysed the data. $\mathrm{XL}, \mathrm{VB}$, and TMO drafted the manuscript. $\mathrm{XL}, \mathrm{EA}, \mathrm{KGI}, \mathrm{KM}, \mathrm{SMM}, \mathrm{VB}$, and TMO interpreted the data and revised the manuscript critically. VB and TMO are joint last authors on this manuscript. $\mathrm{XL}$ is the guarantor.

Funding: XL, SM, and TMO are supported by the National Institute of Mental Health (NIMH) (R01MH104468). XL is also supported by the Danish Council for Independent Research (DFF-5053-00156B) SMM has received research grant support from Sage Therapeutics and Janssen to the University of North Carolina. EA, KGI, KM, and TMO are supported by IPSYCH, the Lundbeck Foundation Initiative for Integrative Psychiatric Research (R155-2014-1724). EA is also supported by a Niels Bohr professorship grant from the Danish National Research Foundation and the Stanley Medical Research Institute. VB has received funding from the Netherlands Organization for Scientific Research (clinical fellow and VENI incentive). The investigators conducted the research independently. The funders had no role in the design and conduct of the study; collection, management, analysis, and interpretation of the data; preparation, review, or approval of the manuscript; or the decision to submit the manuscript for publication

Competing interests: All authors have completed the ICMJE uniform disclosure form at www.icmje.org/coi_disclosure.pdf (available on request from the corresponding author) and declare: support for the submitted work as described above; no financial relationships with any organisations that might have an interest in the submitted work in the previous three years; no other relationships or activities that could appear to have influenced the submitted work

Ethical approval: The study was approved by the Danish Data Protection Agency. By Danish law, no informed consent is required for a register based study using anonymised data.

Transparency: The lead author (the manuscript's guarantor) affirms that this manuscript is an honest, accurate, and transparent account of the study being reported; that no important aspects of the study have been omitted; and that any discrepancies from the study as planned (and, if relevant, registered) have been explained.

Data sharing: No additional data available.

This is an Open Access article distributed in accordance with the Creative Commons Attribution Non Commercial (CC BY-NC 4.0) license, which permits others to distribute, remix, adapt, build upon this work non-commercially, and license their derivative works on different terms, provided the original work is properly cited and the use is noncommercial. See: http://creativecommons.org/licenses/by-nc/4.0/.

1 Jimenez-Solem E, Andersen JT, Petersen M, et al. Prevalence of antidepressant use during pregnancy in Denmark, a nation-wide cohort study. PLoS One 2013;8:e63034. doi:10.1371/journal.pone.0063034

2 Malm H, Brown AS, Gissler M, et al. Gestational Exposure to

Selective Serotonin Reuptake Inhibitors and Offspring Psychiatric Disorders: A National Register-Based Study. J Am Acad Child Adolesc Psychiatry 2016:55:359-66. doi:10.1016/j.jaac.2016.02.013

3 Viktorin A, Uher R, Reichenberg A, Levine SZ, Sandin S. Autism risk following antidepressant medication during pregnancy. Psychol Med 2017;May 22:1-10. doi:10.1017/S0033291717001301

4 Zoega H, Kieler H, Nørgaard M, et al. Use of SSRI and SNRI Antidepressants during Pregnancy: A Population-Based Study from Denmark, Iceland, Norway and Sweden. PLoS One 2015;10:e0144474. doi:10.1371/journal.pone.0144474

5 Petersen I, Gilbert RE, Evans SJ, Man SL, Nazareth I. Pregnancy as a major determinant for discontinuation of antidepressants: an analysis of data from The Health Improvement Network. / Clin Psychiatry 2011;72:979-85. doi:10.4088/JCP.10m06090blu 6 Huybrechts KF, Palmsten K, Mogun H, et al. National trends in antidepressant medication treatment among publicly insured pregnant women. Gen Hosp Psychiatry 2013;35:265-71. doi:10.1016/j.genhosppsych.2012.12.010

7 Brown HK, Ray JG, Wilton AS, Lunsky Y, Gomes T, Vigod SN. Association Between Serotonergic Antidepressant Use During Pregnancy and Autism Spectrum Disorder in Children. JAMA 2017;317:1544-52. doi:10.1001/jama.2017.3415

8 Huybrechts KF, Palmsten K, Avorn J, et al. Antidepressant use in pregnancy and the risk of cardiac defects. N Engl J Med 2014;370:2397-407. doi:10.1056/NEJMoa1312828

9 Hviid A, Melbye M, Pasternak B. Use of selective serotonin reuptake inhibitors during pregnancy and risk of autism. N Engl J Med 2013;369:2406-15. doi:10.1056/NEJMoa1301449

10 Stephansson O, Kieler H, Haglund B, et al. Selective serotonin reuptake inhibitors during pregnancy and risk of stillbirth and infant mortality. JAMA 2013;309:48-54. doi:10.1001/jama.2012.153812

11 Viktorin A, Lichtenstein P, Lundholm C, et al. Selective serotonin re-uptake inhibitor use during pregnancy: association with offspring birth size and gestational age. Int J Epidemiol 2016;45:170-7. doi:10.1093/ije/dyv351

12 Clements CC, Castro VM, Blumenthal SR, et al. Prenatal antidepressant exposure is associated with risk for attention-deficit hyperactivity disorder but not autism spectrum disorder in a large health system. Mol Psychiatry 2015;20:727-34. doi:10.1038/mp.2014.90

13 Boukhris T, Sheehy O, Mottron L, Bérard A. Antidepressant Use During Pregnancy and the Risk of Autism Spectrum Disorder in Children. JAMA Pediatr 2016;170:117-24. doi:10.1001/ jamapediatrics.2015.3356

14 Gidaya NB, Lee BK, Burstyn I, Yudell M, Mortensen EL, Newschaffer CJ. In utero exposure to selective serotonin reuptake inhibitors and risk for autism spectrum disorder. / Autism Dev Disord 2014:44: 2558-67. doi:10.1007/s10803-014-2128-4

15 Rai D, Lee BK, Dalman C, Golding J, Lewis G, Magnusson C. Parental depression, maternal antidepressant use during pregnancy, and risk of autism spectrum disorders: population based case-control study. BMJ 2013;346:f2059. doi:10.1136/bmj.f2059 
16 Croen LA, Grether JK, Yoshida CK, Odouli R, Hendrick V. Antidepressant use during pregnancy and childhood autism spectrum disorders. Arch Gen Psychiatry 2011;68:1104-12. doi:10.1001/archgenpsychiatry.2011.73

17 Brown AS, Gyllenberg D, Malm H, et al. Association of Selective Serotonin Reuptake Inhibitor Exposure During Pregnancy With Speech, Scholastic, and Motor Disorders in Offspring. JAMA Psychiatry 2016;73:1163-70. doi:10.1001/ jamapsychiatry.2016.2594

18 Sørensen MJ, Grønborg TK, Christensen J, et al. Antidepressant exposure in pregnancy and risk of autism spectrum disorders. Clin Epidemiol 2013;5:449-59. doi:10.2147/CLEP.S53009

19 Sujan AC, Rickert ME, Öberg AS, et al. Associations of Maternal Antidepressant Use During the First Trimester of Pregnancy With Preterm Birth, Small for Gestational Age, Autism Spectrum Disorder, and Attention-Deficit/Hyperactivity Disorder in Offspring. JAMA 2017;317:1553-62. doi:10.1001/jama.2017.3413

20 Laugesen K, Olsen MS, Telén Andersen AB, Frøslev T, Sørensen HT. In utero exposure to antidepressant drugs and risk of attention deficit hyperactivity disorder: a nationwide Danish cohort study. BMJ Open 2013;3:e003507. doi:10.1136/ bmjopen-2013-003507

21 Figueroa R. Use of antidepressants during pregnancy and risk of attention-deficit/hyperactivity disorder in the offspring. / Dev Behav Pediatr 2010;31:641-8. doi:10.1097/ DBP.0b013e3181e5ac93

22 Maciag D, Simpson KL, Coppinger D, et al. Neonatal antidepressant exposure has lasting effects on behavior and serotonin circuitry. Neuropsychopharmacology 2006:31:47-57.

23 Glover ME, Pugh PC, Jackson NL, et al. Early-life exposure to the SSRI paroxetine exacerbates depression-like behavior in anxiety/depression-prone rats. Neuroscience 2015;284:775-97. doi:10.1016/i.neuroscience.2014.10.044

24 Videman M, Tokariev A, Saikkonen H, et al. Newborn Brain Function Is Affected by Fetal Exposure to Maternal Serotonin Reuptake Inhibitors. Cereb Cortex 2017;27:3208-16.

25 Sprowles JL, Hufgard JR, Gutierrez A, et al. Perinatal exposure to the selective serotonin reuptake inhibitor citalopram alters spatial learning and memory, anxiety, depression, and startle in SpragueDawley rats. Int J Dev Neurosci 2016;54:39-52. doi:10.1016/j. ijdevneu.2016.08.007

26 Oberlander TF, Gingrich JA, Ansorge MS. Sustained neurobehavioral effects of exposure to SSRI antidepressants during development: molecular to clinical evidence. Clin Pharmacol Ther 2009;86:672-7. doi:10.1038/clpt.2009.201

27 Ciraulo DA, Shader RI, Greenblatt DJ. Clinical pharmacology and therapeutics of antidepressants. In: Ciraulo DA, Shader RI, eds. Pharmacotherapy of depression . Springer, 2011:34doi:10.1007/978-1-60327-435-7 2

28 Pedersen CB. The Danish Civil Registration System. Scand J Public Health 2011;39(Suppl):22-5. doi: $10.1177 / 1403494810387965$

29 Knudsen LB, Olsen J. The Danish Medical Birth Registry. Dan Med Bull 1998;45:320-3.

30 Andersen TF, Madsen M, Jørgensen J, Mellemkjoer L, Olsen JH. The Danish National Hospital Register. A valuable source of data for modern health sciences. Dan Med Bull 1999:46:263-8.

31 Kildemoes HW, Sørensen HT, Hallas J. The Danish National Prescription Registry. Scand J Public Health 2011;39(Suppl):38-41. doi:10.1177/1403494810394717

32 Mors O, Perto GP, Mortensen PB. The Danish Psychiatric Central Research Register. Scand J Public Health 2011;39(Suppl):54-7. doi:10.1177/1403494810395825

33 Petersson F, Baadsgaard M, Thygesen LC. Danish registers on personal labour market affiliation. Scand J Public Health 2011:39(Suppl):95-8. doi:10.1177/1403494811408483
34 Royston P, White IR. Multiple Imputation by Chained Equations (MICE): Implementation in Stata. J Stat Softw 2011;45:1-20. doi:10.18637/jss.v045.i04

35 Cole SR, Hernán MA. Adjusted survival curves with inverse probability weights. Comput Methods Programs Biomed 2004;75:45-9. doi:10.1016/j.cmpb.2003.10.004

36 Rockhill B, Newman B, Weinberg C. Use and misuse of population attributable fractions. Am J Public Health 1998;88:15-9. doi:10.2105/AJPH.88.1.15

37 Smith GD. Assessing intrauterine influences on offspring health outcomes: can epidemiological studies yield robust findings? Basic Clin Pharmacol Toxicol 2008;102:245-56. doi:10.1111/ j.1742-7843.2007.00191.x

38 Dean K, Stevens H, Mortensen PB, Murray RM, Walsh E, Pedersen CB. Full spectrum of psychiatric outcomes among offspring with parental history of mental disorder. Arch Gen Psychiatry 2010;67:822-9. doi:10.1001/archgenpsychiatry.2010.86

39 McLearn KT, Minkovitz CS, Strobino DM, Marks E, Hou W. Maternal depressive symptoms at 2 to 4 months post partum and early parenting practices. Arch Pediatr Adolesc Med 2006;160:279-84. doi:10.1001/archpedi.160.3.279

40 O'Connor TG, Ben-Shlomo Y, Heron J, Golding J, Adams D, Glover V. Prenatal anxiety predicts individual differences in cortisol in preadolescent children. Biol Psychiatry 2005;58:211-7. doi:10.1016/j. biopsych.2005.03.032

41 Ornoy A. Neurobehavioral risks of SSRIs in pregnancy: Comparing human and animal data. Reprod Toxicol 2017;508906238(17)30135-1.

42 Nulman I, Rovet J, Stewart DE, et al. Child development following exposure to tricyclic antidepressants or fluoxetine throughout fetal life: a prospective, controlled study. Am J Psychiatry 2002;159:188995. doi:10.1176/appi.ajp.159.11.1889

43 Vigod SN, Wilson CA, Howard LM. Depression in pregnancy. BMJ 2016;352:i1547. doi:10.1136/bmj.i1547

44 Dansk Selskab for Almen Medicin. Klinisk vejledning for almen praksis: Unipolar depression diagnostik og behandling. 2010. http:// www.dsam.dk/files/9/depression_med_links.pdf.

45 Frisell T, Öberg S, Kuja-Halkola R, Sjölander A. Sibling comparison designs: bias from non-shared confounders and measurement error. Epidemiology 2012;23:713-20. doi:10.1097/EDE.0b013e31825fa230

46 Ansorge MS, Zhou M, Lira A, Hen R, Gingrich JA. Early-life blockade of the 5 -HT transporter alters emotional behavior in adult mice. Science 2004:306:879-81. doi:10.1126/science.1101678

47 Bonari L, Pinto N, Ahn E, Einarson A, Steiner M, Koren G. Perinatal risks of untreated depression during pregnancy. Can J Psychiatry 2004:49:726-35. doi:10.1177/070674370404901103

48 Yonkers KA, Wisner KL, Stewart DE, et al. The management of depression during pregnancy: a report from the American Psychiatric Association and the American College of Obstetricians and Gynecologists. Obstet Gynecol 2009;114:703-13. doi:10.1097/ AOG.0b013e3181ba0632

49 Olesen C, Søndergaard C, Thrane N, Nielsen GL, de Jong-van den Berg L, Olsen J: EuroMAP Group. Do pregnant women report use of dispensed medications? Epidemiology 2001;12:497-501. doi:10.1097/00001648-200109000-00006

50 Lahon K, Shetty HM, Paramel A, et al. A retrospective drug utilization study of antidepressants in the psychiatric unit of a tertiary care hospital. J Clin Diagn Res 2011;5:1069-75.

51 Pedersen CB, Mors O, Bertelsen A, et al. A comprehensive nationwide study of the incidence rate and lifetime risk for treated mental disorders. JAMA Psychiatry 2014;71:573-81. doi:10.1001/ jamapsychiatry.2014.16

\section{Supplementary materials}

